Mathematical Modelling and Analysis

Volume 19 Number 1, February 2014, 66-74

http://dx.doi.org/10.3846/13926292.2014.893262

(C) Vilnius Gediminas Technical University, 2014
Publisher: Taylor\&Francis and VGTU

http://www.tandfonline.com/TMMA

Print ISSN: 1392-6292

Online ISSN: 1648-3510

\title{
Limit Theorems for Twists of $L$-Functions of Elliptic Curves. IV
}

\author{
Virginija Garbaliauskienè $\dot{x}^{a}$ and Antanas Laurinčikas ${ }^{b}$ \\ ${ }^{a}$ Šiauliai University \\ P. Višinskio str. 19, LT-77156 Šiauliai, Lithuania \\ ${ }^{b}$ Vilnius University \\ Naugarduko str. 24, LT-03225 Vilnius, Lithuania \\ E-mail(corresp.): virginija@fm.su.lt \\ E-mail: antanas.laurincikas@mif.vu.lt
}

Received June 29, 2013; revised December 13, 2013; published online February 20, 2014

\begin{abstract}
In this paper, we prove a multidimensional limit theorem for moduli of twists of $L$-functions of elliptic curves. The limit measure in this theorem is defined by the characteristic transforms.

Keywords: Dirichlet character, elliptic curve, $L$-function of elliptic curve, twist of $L$ function of elliptic curve, weak convergence.
\end{abstract}

AMS Subject Classification: 11M41; 44K15.

\section{Introduction}

In [3], we have obtained a limit theorem for the modulus of twisted with Dirichlet character $L$-functions of elliptic curves with an increasing modulus of the character. Let $E$ be an elliptic curve over the field of rational numbers given by the Weierstrass equation

$$
y^{2}=x^{3}+a x+b, \quad a, b \in \mathbb{Z}
$$

with discriminant $\Delta=-16\left(4 a^{3}+27 b^{2}\right) \neq 0$. For each prime number $p$, denote by $E_{p}$ the reduction of the curve $E$ modulo $p$ which is a curve over the finite field $\mathbb{F}_{p}$, and define $\lambda(p)$ by the equality

$$
\left|E\left(\mathbb{F}_{p}\right)\right|=p+1-\lambda(p),
$$

where $\left|E\left(\mathbb{F}_{p}\right)\right|$ is the number of points of $E_{p}$. The $L$-function $L_{E}(s), s=\sigma+i t$, of the elliptic curve $E$ is defined, for $\sigma>\frac{3}{2}$, by the product

$$
L_{E}(s)=\prod_{p \mid \Delta}\left(1-\frac{\lambda(p)}{p^{s}}\right)^{-1} \prod_{p \nmid \Delta}\left(1-\frac{\lambda(p)}{p^{s}}+\frac{1}{p^{2 s-1}}\right)^{-1}
$$


and continues analytically to the whole complex plane.

Let $\chi$ be a Dirichlet character modulo $q$. The twist $L_{E}(s, \chi)$ with character $\chi$ for the function $L_{E}(s)$ is defined, for $\sigma>\frac{3}{2}$, by

$$
L_{E}(s, \chi)=\prod_{p \mid \Delta}\left(1-\frac{\lambda(p) \chi(p)}{p^{s}}\right)^{-1} \prod_{p \nmid \Delta}\left(1-\frac{\lambda(p) \chi(p)}{p^{s}}+\frac{\chi^{2}(p)}{p^{2 s-1}}\right)^{-1}
$$

and is analytically continued to an entire function.

Suppose that the modulus $q$ of the character $\chi$ is a prime number, denote, as usual, by $\chi_{0}$ the principal character modulo $q$, and, for $Q \geq 2$, define

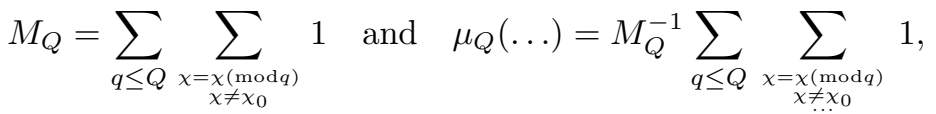

where in place of dots we will write a condition satisfied by a pair $(q, \chi(\bmod q))$. Moreover, denote by $\mathcal{B}(X)$ the Borel $\sigma$-field of the space $X$. Then, in [3], for $\sigma>\frac{3}{2}$, we have proved a limit theorem on the weak convergence of the measure

$$
\mu_{Q}\left(\left|L_{E}(s, \chi)\right| \in A\right), \quad A \in \mathcal{B}(\mathbb{R})
$$

as $Q \rightarrow \infty$. The limit measure $P$ in that theorem is defined by its characteristic transforms

$$
w_{k}(\tau)=\int_{\mathbb{R} \backslash\{0\}}|x|^{i \tau} \operatorname{sgn}^{k} \mathrm{~d} P=\sum_{m=1}^{\infty} \frac{a_{\tau}(m) b_{\tau}(m)}{m^{2 \sigma}}, \quad \tau \in \mathbb{R}, k=0,1,
$$

where $a_{\tau}(m)$ and $b_{\tau}(m)$ are certain explicitly given multiplicative functions.

We note that the first theorems of the above type were obtained by P. Elliott [1] and [2], and E. Stankus [5] for Dirichlet $L$-functions.

The aim of this note is to prove a joint limit theorem for the moduli of the twists of $L$-functions of elliptic curves. For $j=1, \ldots, r$, let $E_{j}$ be an elliptic curve given by the Weierstrass equation

$$
y^{2}=x^{3}+a_{j} x+b_{j}, \quad a_{j}, b_{j} \in \mathbb{Z}
$$

with non-zero discriminant $\Delta_{j}=-16\left(4 a_{j}^{3}+27 b_{j}^{2}\right)$. Similarly as above, we define the quantities $\lambda_{j}(p)$, and the twist

$$
L_{E_{j}}(s, \chi)=\prod_{p \mid \Delta_{j}}\left(1-\frac{\lambda_{j}(p) \chi(p)}{p^{s}}\right)^{-1} \prod_{p \nmid \Delta_{j}}\left(1-\frac{\lambda_{j}(p) \chi(p)}{p^{s}}+\frac{\chi^{2}(p)}{p^{2 s-1}}\right)^{-1}, \quad \sigma>\frac{3}{2} .
$$

This, for $\sigma>\frac{3}{2}$, can be rewritten in the form

$$
\begin{aligned}
L_{E_{j}}(s, \chi)= & \prod_{p \mid \Delta_{j}}\left(1-\frac{\lambda_{j}(p) \chi(p)}{p^{s}}\right)^{-1} \prod_{p \nmid \Delta_{j}}\left(1-\frac{\alpha_{j}(p) \chi(p)}{p^{s}}\right)^{-1} \\
& \times\left(1-\frac{\beta_{j}(p) \chi(p)}{p^{s}}\right)^{-1},
\end{aligned}
$$


where $\alpha_{j}(p)$ and $\beta_{j}(p)$ are conjugate complex numbers such that $\alpha_{j}(p)+\beta_{j}(p)=$ $\lambda_{j}(p)$ and $\alpha_{j}(p) \beta_{j}(p)=p$. For brevity, let $\eta=\eta(\tau)=\frac{i \tau}{2}, \tau \in \mathbb{R}$, and, for primes $p$ and $k \in \mathbb{N}$, we set

$$
d_{\tau}\left(p^{k}\right)=\frac{\eta(\eta+1) \cdots(\eta+k-1)}{k !} .
$$

For $j=1, \ldots, r, p \nmid \Delta_{j}$ and $k \in \mathbb{N}$, we define

$$
\begin{aligned}
& a_{j ; \tau}\left(p^{k}\right)=\sum_{l=0}^{k} d_{\tau}\left(p^{l}\right) \alpha_{j}^{l}(p) d_{\tau}\left(p^{k-l}\right) \beta_{j}^{k-l}(p), \\
& b_{j ; \tau}\left(p^{k}\right)=\sum_{l=0}^{k} d_{\tau}\left(p^{l}\right) \bar{\alpha}_{j}^{l}(p) d_{\tau}\left(p^{k-l}\right) \bar{\beta}_{j}^{k-l}(p),
\end{aligned}
$$

where $\bar{\alpha}_{j}(p)$ and $\bar{\beta}_{j}(p)$ denote the conjugates of $\alpha_{j}(p)$ and $\beta_{j}(p)$, respectively. For $j=1, \ldots, r, p \mid \Delta_{j}$ and $k \in \mathbb{N}$, we define

$$
a_{j ; \tau}\left(p^{k}\right)=b_{j ; \tau}\left(p^{k}\right)=d_{\tau}\left(p^{k}\right) \lambda_{j}^{k}(p) .
$$

Now, for $j=1, \ldots, r$ and $m \in \mathbb{N}$, define

$$
a_{j ; \tau}(m)=\prod_{p^{l} \| m} a_{j ; \tau}\left(p^{l}\right), \quad b_{j ; \tau}(m)=\prod_{p^{l} \| m} b_{j ; \tau}\left(p^{l}\right)
$$

where $p^{l} \| m$ means that $p^{l} \mid m$ but $p^{l+1} \nmid m$. Thus, $a_{j ; \tau}(m)$ and $b_{j ; \tau}(m)$ are multiplicative functions.

Now, on $\left(\mathbb{R}^{r}, \mathcal{B}\left(\mathbb{R}^{r}\right)\right)$, we will define a certain probability measure $P^{(r)}$. For this, we will use the characteristic transforms. Let $P$ be a probability measure on $\left(\mathbb{R}^{r}, \mathcal{B}\left(\mathbb{R}^{r}\right)\right)$. Denote by $P_{j}, j=1, \ldots, r, P_{j_{1}, j_{2}}, j_{2}>j_{1}=1, \ldots, r-1$, $\ldots, P_{1, \ldots, j-1, j+1, \ldots, r}, j=1, \ldots, r$, the one-dimensional, two-dimensional, $\ldots$, $(r-1)$-dimensional marginal measures of $P$, i.e.,

$$
\begin{aligned}
& P_{j}(A)=P(\underbrace{\mathbb{R} \times \cdots \mathbb{R}}_{j-1} \times A \times \mathbb{R} \times \cdots \times \mathbb{R}), \quad A \in \mathcal{B}(\mathbb{R}), j=1, \ldots, r, \\
& P_{j_{1}, j_{2}}\left(A_{1} \times A_{2}\right)=P(\underbrace{\underbrace{\mathbb{R} \times \cdots \mathbb{R}}_{j_{1}-1} \times A_{1} \times \mathbb{R} \times \cdots \times \mathbb{R}}_{j_{2}-1} \times A_{2} \times \mathbb{R} \times \cdots \times \mathbb{R}), \\
& A_{1}, A_{2} \in \mathcal{B}(\mathbb{R}), j_{2}>j_{1}=1, \ldots, r-1, \\
& P_{1, \ldots, j-1, j+1, \ldots, r}\left(A_{1} \times \cdots \times A_{j-1} \times A_{j+1} \times \cdots \times A_{r}\right) \\
& =P\left(A_{1} \times \cdots \times A_{j-1} \times \mathbb{R} \times A_{j+1} \times \cdots \times A_{r}\right), \\
& A_{1}, \ldots, A_{j-1}, A_{j+1}, \ldots, A_{r} \in \mathcal{B}(\mathbb{R}), j=1, \ldots, r .
\end{aligned}
$$


Then the functions

$$
\begin{aligned}
& w_{k}(\tau)=\int_{\mathbb{R} \backslash\{0\}}|x|^{i \tau} \operatorname{sgn}^{k} x \mathrm{~d} P_{j}, \quad \tau \in \mathbb{R}, k=0,1, j=1, \ldots, r, \\
& w_{k_{1}, k_{2}}\left(\tau_{1}, \tau_{2}\right)=\int_{\mathbb{R} \backslash\{0\}} \int_{\mathbb{R} \backslash\{0\}}\left(\left|x_{1}\right|^{i \tau_{1}} \operatorname{sgn}^{k_{1}} x_{1}\right)\left(\left|x_{2}\right|^{i \tau_{2}} \operatorname{sgn}^{k_{2}} x_{2}\right) \mathrm{d} P_{j_{1}, j_{2}}, \\
& \tau_{1}, \tau_{2} \in \mathbb{R}, k_{1}, k_{2}=0,1, j_{2}>j_{1}=1, \ldots, r, \\
& w_{k_{1}, \ldots, k_{j-1}, k_{j+1}, \ldots, k_{r}}\left(\tau_{1}, \ldots, \tau_{j-1}, \tau_{j+1}, \ldots, \tau_{r}\right) \\
& =\int_{\mathbb{R} \backslash\{0\}} \cdots \int_{\mathbb{R} \backslash\{0\}}\left(\left|x_{1}\right|^{i \tau_{1}} \operatorname{sgn}^{k_{1}} x_{1}\right) \cdots\left(\left|x_{j-1}\right|^{i \tau_{j-1}} \operatorname{sgn}^{k_{j-1}} x_{j-1}\right) \\
& \left(\left|x_{j+1}\right|^{i \tau_{j+1}} \operatorname{sgn}^{k_{j+1}} x_{j+1}\right) \cdots\left(\left|x_{r}\right|^{i \tau_{r}} \operatorname{sgn}^{k_{r}} x_{r}\right) \mathrm{d} P_{1, \ldots, j-1, j+1, \ldots, r}, \\
& \tau_{1}, \ldots, \tau_{j-1}, \tau_{j+1}, \ldots, \tau_{r} \in \mathbb{R}, k_{1}, \ldots, k_{j-1}, k_{j+1}, \ldots, k_{r}=0,1, j=1, \ldots, r, \\
& w_{k_{1}, \ldots, k_{r}}\left(\tau_{1}, \ldots, \tau_{r}\right)=\int_{\mathbb{R} \backslash\{0\}} \ldots \int_{\mathbb{R} \backslash\{0\}}\left(\left|x_{1}\right|^{i \tau_{1}} \operatorname{sgn}^{k_{1}} x_{1}\right) \cdots\left(\left|x_{r}\right|^{i \tau_{r}} \operatorname{sgn}^{k_{r}} x_{r}\right) \mathrm{d} P, \\
& \tau_{1}, \ldots, \tau_{r} \in \mathbb{R}, k_{1}, \ldots, k_{r}=0,1
\end{aligned}
$$

are called the characteristic transforms of the measure $P$. The characteristic transforms of multidimensional distribution functions were introduced in [4]. It is easily seen that the results of [4] remain valid when distribution functions are replaced by probability measures. Thus, we have that the measure $P$ on $\left(\mathbb{R}^{r}, \mathcal{B}\left(\mathbb{R}^{r}\right)\right)$ is uniquely determined by its characteristic transforms $\left\{w_{k}(\tau), w_{k_{1}, k_{2}}\left(\tau_{1}, \tau_{2}\right), \ldots, w_{k_{1}, \ldots, k_{j-1}, k_{j+1}, \ldots, k_{r}}\left(\tau_{1}, \ldots, \tau_{j-1}, \tau_{j+1}, \ldots, \tau_{r}\right)\right.$, $\left.w_{k_{1}, \ldots, k_{r}}\left(\tau_{1}, \ldots, \tau_{r}\right)\right\}$.

Now let $P^{(r)}$ be a probability measure on $\left(\mathbb{R}^{r}, \mathcal{B}\left(\mathbb{R}^{r}\right)\right)$ given by the following characteristic transforms:

$$
\begin{aligned}
& w_{k}(\tau)=\sum_{m=1}^{\infty} \frac{a_{j ; \tau}(m) b_{j ; \tau}(m)}{m^{2 \sigma_{j}}}, \quad j=1, \ldots, r, \\
& w_{k_{1}, k_{2}}\left(\tau_{1}, \tau_{2}\right)=\sum_{m=1}^{\infty} \sum_{m_{1} m_{2}=m} \frac{a_{j_{1} ; \tau_{1}}\left(m_{1}\right) a_{j_{2} ; \tau_{2}}\left(m_{2}\right)}{m_{1}^{s_{1}} m_{2}^{s_{2}}} \sum_{n_{1}} \frac{b_{n_{1} ; \tau_{1}}\left(n_{1}\right) b_{j_{2} ; \tau_{2}}\left(n_{2}\right)}{n_{1}^{\bar{s}_{1}} n_{2}^{\bar{s}_{2}}} \\
& j_{2}>j_{1}=1, \ldots, r-1, \\
& w_{k_{1}, \ldots, k_{j-1}, k_{j+1}, \ldots, k_{r}}\left(\tau_{1}, \ldots, \tau_{j-1}, \tau_{j+1}, \ldots, \tau_{r}\right) \\
& =\sum_{m=1}^{\infty} \sum_{m_{1} \ldots m_{j-1}} \frac{a_{m_{j+1} \ldots \tau_{1}}\left(m_{1}\right) \cdots a_{r-1 ; \tau_{j-1}=m}\left(m_{j-1}\right) a_{j+1 ; \tau_{j+1}}\left(m_{j+1}\right) \cdots a_{r ; \tau_{r}}\left(m_{r}\right)}{m_{1}^{s_{1}} \cdots m_{j-1}^{s_{j-1}} m_{j+1}^{s_{j+1} \cdots m_{r}^{s_{r}}}} \\
& \times \sum_{n_{1} \cdots n_{j-1}} \sum_{n_{j+1} \cdots n_{r}=m} \frac{b_{1 ; \tau_{1}}\left(n_{1}\right) \cdots b_{j-1 ; \tau_{j-1}}\left(n_{j-1}\right) b_{j+1 ; \tau_{j+1}}\left(n_{j+1}\right) \cdots b_{r ; \tau_{r}}\left(n_{r}\right)}{n_{1}^{\bar{s}_{1}} \cdots n_{j-1}^{\bar{s}_{j-1}} n_{j+1}^{\bar{s}_{j+1}} \cdots n_{r}^{\bar{s}_{r}}}, \\
& j=1, \ldots, r,
\end{aligned}
$$




$$
\begin{aligned}
w_{k_{1}, \ldots, k_{r}}\left(\tau_{1}, \ldots, \tau_{r}\right)= & \sum_{m=1}^{\infty} \sum_{m_{1} \cdots m_{r}=m} \frac{a_{1 ; \tau_{1}}\left(m_{1}\right) \cdots a_{r ; \tau_{r}}\left(m_{r}\right)}{m_{1}^{s_{1}} \cdots m_{r}^{s_{r}}} \\
& \times \sum_{n_{1} \cdots n_{r}=m} \frac{b_{1 ; \tau_{1}}\left(n_{1}\right) \cdots b_{r ; \tau_{r}}\left(n_{r}\right)}{n_{1}^{\bar{s}_{1}} \cdots n_{r}^{\bar{s}_{r}}} .
\end{aligned}
$$

Here $s_{j}=\sigma_{j}+i t_{j}$, and $\sigma_{j}>\frac{3}{2}, j=1, \ldots, r$.

For $A \in \mathcal{B}\left(\mathbb{R}^{r}\right)$, define

$$
P_{Q}(A)=\mu_{Q}\left(\left(\left|L_{E_{1}}\left(s_{1}, \chi\right)\right|, \ldots,\left|L_{E_{r}}\left(s_{r}, \chi\right)\right|\right) \in A\right) .
$$

Theorem 1. Suppose that $\min _{1 \leq j \leq r} \sigma_{j}>\frac{3}{2}$. Then $P_{Q}$ converges weakly to the probability measure $P^{(r)}$ as $Q \rightarrow \infty$.

For the proof of Theorem 1, we will apply the method of characteristic transforms.

\section{Characteristic Transforms of the Measure $P_{Q}$}

In this section, we derive the formulae for the characteristic transforms $\left\{w_{j ; Q}(\tau), w_{j_{1}, j_{2} ; Q}\left(\tau_{1}, \tau_{2}\right), \ldots, w_{1, \ldots, j-1, j+1, \ldots, r ; Q}\left(\tau_{1}, \ldots, \tau_{j-1}, \tau_{j+1}, \ldots, \tau_{r}\right)\right.$, $\left.w_{Q}\left(\tau_{1}, \ldots, \tau_{r}\right)\right\}$ of the measure $P_{Q}$. Since $P_{Q}$ is defined by means of the moduli of the twists $L_{E_{j}}(s, \chi), j=1, \ldots, r$, its characteristic transforms do not depend on $k, k_{1}, k_{2} ; \ldots ; k_{1}, \ldots, k_{r}$. The definitions of $P_{Q}$ and of characteristic transforms imply that

$$
\begin{aligned}
& w_{j ; Q}(\tau)=\frac{1}{M_{Q}} \sum_{q \leq Q} \sum_{\substack{x=\chi(\bmod q) \\
\chi \neq \chi_{0}}}\left|L_{E_{j}}(s, \chi)\right|^{i \tau}, \quad j=1, \ldots, r, \\
& w_{j_{1}, j_{2} ; Q}\left(\tau_{1}, \tau_{2}\right)=\frac{1}{M_{Q}} \sum_{q \leq Q} \sum_{\substack{x=\chi(\bmod q) \\
\chi \neq \chi_{0}}}\left|L_{E_{j_{1}}}\left(s_{1}, \chi\right)\right|^{i \tau_{1}}\left|L_{E_{j_{2}}}\left(s_{2}, \chi\right)\right|^{i \tau_{2}}, \\
& j_{2}>j_{1}=1, \ldots, r-1, \\
& w_{1, \ldots, j-1, j+1, \ldots, r ; Q}\left(\tau_{1}, \ldots, \tau_{j-1}, \tau_{j+1}, \ldots, \tau_{r}\right) \\
& =\frac{1}{M_{Q}} \sum_{q \leq Q} \sum_{\substack{x=\chi(\bmod q) \\
\chi \neq \chi_{0}}}\left|L_{E_{1}}\left(s_{1}, \chi\right)\right|^{i \tau_{1}}, \ldots,\left|L_{E_{j-1}}\left(s_{j-1}, \chi\right)\right|^{i \tau_{j-1}} \\
& \times\left|L_{E_{j+1}}\left(s_{j+1}, \chi\right)\right|^{i \tau_{j+1}} \cdots\left|L_{E_{r}}\left(s_{r}, \chi\right)\right|^{i \tau_{r}}, \quad j=1, \ldots, r, \\
& w_{Q}\left(\tau_{1}, \ldots, \tau_{r}\right)=\frac{1}{M_{Q}} \sum_{q \leq Q} \sum_{\substack{\alpha=\chi(\bmod q) \\
\chi \neq \chi_{0}}}\left|L_{E_{1}}\left(s_{1}, \chi\right)\right|^{i \tau_{1}} \cdots\left|L_{E_{r}}\left(s_{r}, \chi\right)\right|^{i \tau_{r}} .
\end{aligned}
$$

Let $\delta$ be a fixed small positive number, and let $R_{j}=\left\{s \in \mathbb{C}: \sigma_{j} \geq \frac{3}{2}+\delta\right\}$. Then, in [3], it was obtained that

$$
w_{j ; Q}(\tau)=\sum_{m=1}^{\infty} \frac{a_{j ; \tau}(m) b_{j ; \tau}(m)}{m^{2 \sigma_{j}}}+\mathrm{o}(1)
$$


uniformly in $|\tau| \leq c$ and $s_{j} \in R_{j}$ with arbitrary $c>0$, as $Q \rightarrow \infty, j=1, \ldots, r$. Therefore, it remains to consider the characteristic transforms $w_{j_{1}, j_{2} ; Q}\left(\tau_{1}, \tau_{2}\right)$, $\ldots, w_{1, \ldots, j-1, j+1, \ldots, r ; Q}\left(\tau_{1}, \ldots, \tau_{j-1}, \tau_{j+1}, \ldots, \tau_{r}\right), w_{Q}\left(\tau_{1}, \ldots, \tau_{r}\right)$.

In [3], it was obtained that, for $s_{j} \in R_{j}$,

$$
\left|L_{E_{j}}\left(s_{j}, \chi\right)\right|^{i \tau}=\sum_{m=1}^{\infty} \frac{\hat{a}_{j ; \tau}(m)}{m^{s_{j}}} \sum_{n=1}^{\infty} \frac{\hat{b}_{j ; \tau}(m)}{n^{\bar{s}_{j}}}
$$

where $\hat{a}_{j ; \tau}(m)$ and $\hat{b}_{j ; \tau}(m)$ are multiplicative functions given, for $p \nmid \Delta_{j}$ and $k \in \mathbb{N}$, by

$$
\begin{aligned}
& \hat{a}_{j ; \tau}\left(p^{k}\right)=\sum_{l=0}^{k} d_{\tau}\left(p^{l}\right) \alpha_{j}^{l}(p) \chi\left(p^{l}\right) d_{\tau}\left(p^{k-l}\right) \beta_{j}^{k-l}(p) \chi\left(p^{k-l}\right) \\
& \hat{b}_{j ; \tau}\left(p^{k}\right)=\sum_{l=0}^{k} d_{\tau}\left(p^{l}\right) \bar{\alpha}_{j}^{l}(p) \bar{\chi}\left(p^{l}\right) d_{\tau}\left(p^{k-l}\right) \bar{\beta}_{j}^{k-l}(p) \bar{\chi}\left(p^{k-l}\right),
\end{aligned}
$$

and, for $p \mid \Delta_{j}, k \in \mathbb{N}$, by

$$
\hat{a}_{j ; \tau}\left(p^{k}\right)=d_{k}\left(p^{k}\right) \lambda_{j}^{k}(p) \chi^{k}(p), \quad \hat{b}_{j ; \tau}\left(p^{k}\right)=d_{\tau}\left(p^{k}\right) \lambda_{j}^{k}(p) \bar{\chi}^{k}(p),
$$

$j=1, \ldots, r$. Moreover, using the Hasse estimate

$$
\left|\lambda_{j}(p)\right| \leq 2 \sqrt{p}
$$

it was observed in [3] that, for $\left|\tau_{j}\right| \leq c$,

$$
\left|\hat{a}_{j ; \tau_{j}}(m)\right| \leq m^{\frac{1}{2}} d^{c_{1}}(m), \quad\left|\hat{b}_{j ; \tau_{j}}(m)\right| \leq m^{\frac{1}{2}} d^{c_{1}}(m),
$$

where $d(m)$ denotes the divisor function, and $c_{1}$ is a positive constant depending only on $c$.

For simplicity, we will consider only the case $w_{1,2 ; Q}\left(\tau_{1}, \tau_{2}\right) \stackrel{\text { def }}{=} w_{Q}\left(\tau_{1}, \tau_{2}\right)$ and $w_{Q}\left(\tau_{1}, \ldots, \tau_{r}\right)$. Other characteristic transforms of the measure $P_{Q}$ are evaluated similarly. From (2.1) and (2.3), we find that, for $s_{j} \in R_{j}$,

$$
\begin{aligned}
& w_{Q}\left(\tau_{1}, \tau_{2}\right)=\frac{1}{M_{Q}} \sum_{q \leq Q} \sum_{\substack{x=\chi(\bmod q) \\
\chi \neq \chi_{0}}} \sum_{m_{1}=1}^{\infty} \frac{\hat{a}_{1 ; \tau_{1}}\left(m_{1}\right)}{m_{1}^{s_{1}}} \\
& \times \sum_{n_{1}=1}^{\infty} \frac{\hat{b}_{1 ; \tau_{1}}\left(n_{1}\right)}{n_{1}^{\bar{s}_{1}}} \sum_{m_{2}=1}^{\infty} \frac{\hat{a}_{2 ; \tau}\left(m_{2}\right)}{m_{2}^{s_{2}}} \sum_{n_{2}=1}^{\infty} \frac{\hat{b}_{2 ; \tau}\left(n_{2}\right)}{n_{2}^{\bar{s}_{2}}}, \\
& w_{Q}\left(\tau_{1}, \ldots, \tau_{r}\right)=\frac{1}{M_{Q}} \sum_{q \leq Q} \sum_{\substack{x=\chi(\bmod q) \\
\chi \neq \chi_{0}}} \sum_{m_{1}=1}^{\infty} \frac{\hat{a}_{1 ; \tau_{1}}\left(m_{1}\right)}{m_{1}^{s_{1}}} \\
& \sum_{n_{1}=1}^{\infty} \frac{\hat{b}_{1 ; \tau_{1}}\left(n_{1}\right)}{n_{1}^{\bar{s}_{1}}} \cdots \sum_{m_{r}=1}^{\infty} \frac{\hat{a}_{r ; \tau_{r}}\left(m_{r}\right)}{m_{r}^{s_{r}}} \sum_{n_{r}=1}^{\infty} \frac{\hat{b}_{r ; \tau_{r}}\left(n_{r}\right)}{n_{r}^{\bar{s}_{r}}},
\end{aligned}
$$

where $\hat{a}_{j ; \tau_{j}}(m)$ and $\hat{b}_{j ; \tau_{j}}(m)$ are multiplicative functions defined by (2.4)-(2.6), and satisfying estimates $(2.7), j=1, \ldots, r$. 


\section{Asymptotics of Characteristic Transforms of $P_{Q}$}

In this section, we consider the characteristic transforms of the measure $P_{Q}$ as $Q \rightarrow \infty$. Let, for brevity, $N=\log Q$. Then the well-known estimate $d(m)=\mathrm{O}_{\varepsilon}\left(m^{\varepsilon}\right)$ with arbitrary $\varepsilon>0$ together with estimates (2.7) shows that, for $\left|\tau_{j}\right| \leq c$ and $s_{j} \in R_{j}$,

$$
\begin{aligned}
& \sum_{m>N} \frac{\hat{a}_{j ; \tau_{j}}(m)}{m^{s_{j}}} \ll \sum_{m>N} \frac{m^{\frac{1}{2}} d^{c_{1}}\left(m_{j}\right)}{m^{\frac{3}{2}+\delta}} \ll_{\varepsilon} \sum_{m>N} \frac{1}{m^{1+\delta-\varepsilon}} \ll_{\varepsilon} N^{-\delta+\varepsilon}, \\
& \sum_{n>N} \frac{\hat{b}_{j ; \tau_{j}}(n)}{n_{j}^{\bar{s}}}=\ll_{\varepsilon} N^{-\delta+\varepsilon},
\end{aligned}
$$

$j=1, \ldots, r$. Since, in view of $(2.7)$, for $\left|\tau_{j}\right| \leq c$ and $s_{j} \in R_{j}$,

$$
\sum_{m \leq N} \frac{\hat{a}_{j ; \tau_{j}}(m)}{m^{s_{j}}}=\mathcal{O}(1) \text { and } \sum_{n \leq N} \frac{\hat{b}_{j ; \tau_{j}}(n)}{n^{\bar{s}_{j}}}=\mathcal{O}(1),
$$

$j=1, \ldots, r$, from this and (2.8) we find that, in the above regions of $\tau_{j}$ and $s_{j}$,

$$
\begin{aligned}
& w_{Q}\left(\tau_{1}, \tau_{2}\right)=\frac{1}{M_{Q}} \sum_{q \leq Q} \sum_{\substack{x=\chi(\bmod q) \\
\chi \neq \neq \chi_{0}}}\left(\sum_{m_{1}=\leq N} \frac{\hat{a}_{1 ; \tau_{1}}\left(m_{1}\right)}{m_{1}^{s_{1}}}\right. \\
& \left.\quad \times \sum_{n_{1} \leq N} \frac{\hat{b}_{1 ; \tau_{1}}\left(n_{1}\right)}{n_{1}^{\bar{s}_{1}}} \sum_{m_{2} \leq N}^{\infty} \frac{\hat{a}_{2 ; \tau_{2}}\left(m_{2}\right)}{m_{2}^{s_{2}}} \sum_{n_{2} \leq N}^{\infty} \frac{\hat{b}_{2 ; \tau_{2}}\left(n_{2}\right)}{n_{2}^{\bar{s}_{2}}}\right)+\mathcal{O}_{\varepsilon}\left(N^{-\delta+\varepsilon}\right), \\
& \ldots \ldots \ldots \ldots \ldots \ldots \ldots \ldots \ldots \ldots \ldots \\
& w_{Q}\left(\tau_{1}, \ldots \ldots, \tau_{r}\right)=\frac{1}{M_{Q}} \sum_{q \leq Q} \sum_{\chi=\chi(\bmod q)}\left(\sum_{m_{1} \leq N} \frac{\hat{a}_{1 ; \tau_{1}}\left(m_{1}\right)}{m_{1}^{s_{1}}}\right. \\
& \left.\quad \times \sum_{n_{1} \leq N} \frac{\hat{b}_{1 ; \tau_{1}}\left(n_{1}\right)}{n_{1}^{\bar{s}_{1}}} \ldots \sum_{m_{r} \leq N}^{\infty} \frac{\hat{a}_{r ; \tau_{r}}\left(m_{r}\right)}{m_{r}^{s_{r}}} \sum_{n_{r} \leq N}^{\infty} \frac{\hat{b}_{r ; \tau_{r}}\left(n_{r}\right)}{n_{r}^{\bar{s}_{r}}}\right)+\mathcal{O}_{\varepsilon}\left(N^{-\delta+\varepsilon}\right) .
\end{aligned}
$$

The multiplicativity of the functions $\hat{a}_{j ; \tau_{j}}(m)$ and $\hat{b}_{j ; \tau_{j}}(m)$, and the complete multiplicativity of the character $\chi$ together with (2.4)-(2.6) and the definition of the multiplicative functions $a_{j ; \tau_{j}}(m)$ and $b_{j ; \tau_{j}}(m)$ show that

$$
\hat{a}_{j ; \tau_{j}}(m)=a_{j ; \tau_{j}}(m) \chi(m), \quad \hat{b}_{j ; \tau_{j}}(m)=b_{j ; \tau_{j}}(m) \bar{\chi}(m),
$$

$j=1, \ldots, r$. Therefore, the main terms on the right-hand sides of (3.1) are of the form

$$
\begin{aligned}
\sum_{m_{1} \leq N} & \frac{a_{1 ; \tau_{1}}\left(m_{1}\right)}{m_{1}^{s_{1}}} \sum_{n_{1} \leq N} \frac{b_{1 ; \tau_{1}}\left(n_{1}\right)}{n_{1}^{\bar{s}_{1}}} \sum_{m_{2} \leq N} \frac{a_{2 ; \tau_{2}}\left(m_{2}\right)}{m_{2}^{s_{2}}} \sum_{n_{2} \leq N} \frac{b_{2 ; \tau_{2}}\left(n_{2}\right)}{n_{2}^{\bar{s}_{2}}} \\
& \times \frac{1}{M_{Q}} \sum_{\substack{q \leq Q \\
q}} \sum_{\substack{x=\chi \bmod q) \\
\chi \neq \neq \chi_{0}}} \chi\left(m_{1} m_{2}\right) \bar{\chi}\left(n_{1} n_{2}\right)
\end{aligned}
$$




$$
\begin{aligned}
& =\sum_{m \leq N^{2}} \sum_{m_{1} m_{2}=m} \frac{a_{1 ; \tau_{1}}\left(m_{1}\right) a_{2 ; \tau_{2}}\left(m_{2}\right)}{m_{1}^{s_{1}} m_{2}^{s_{2}}} \sum_{n \leq N^{2}} \sum_{n_{1} n_{2}=n} \frac{b_{1 ; \tau_{1}}\left(n_{1}\right) b_{2 ; \tau_{2}}\left(n_{2}\right)}{n_{1}^{\bar{s}_{1}} n_{2}^{\bar{s}_{2}}} \\
& \times \frac{1}{M_{Q}} \sum_{q \leq Q} \sum_{\substack{\chi=\chi(\bmod q) \\
\chi \neq \chi_{0}}} \chi(m) \bar{\chi}(n), \\
& \sum_{m_{1} \leq N} \frac{a_{1 ; \tau_{1}}\left(m_{1}\right)}{m_{1}^{s_{1}}} \sum_{n_{1} \leq N} \frac{b_{1 ; \tau_{1}}\left(n_{1}\right)}{n_{1}^{\bar{s}_{1}}} \cdots \sum_{m_{r} \leq N} \frac{a_{r ; \tau_{r}}\left(m_{r}\right)}{m_{r}^{s_{r}}} \sum_{n_{r} \leq N} \frac{b_{r ; \tau_{r}}\left(n_{r}\right)}{n_{r}^{\bar{s}_{r}}} \\
& \times \frac{1}{M_{Q}} \sum_{q \leq Q} \sum_{\substack{\chi=\chi(\bmod q) \\
\chi \neq \chi_{0}}} \chi\left(m_{1} \cdots m_{r}\right) \bar{\chi}\left(n_{1} \cdots n_{r}\right) \\
& =\sum_{m \leq N^{r}} \sum_{m_{1} \cdots m_{r}=m} \frac{a_{1 ; \tau_{1}}\left(m_{1}\right) \cdots a_{r ; \tau_{r}}\left(m_{r}\right)}{m_{1}^{s_{1}} \cdots m_{r}^{s_{r}}} \sum_{n \leq N^{r}} \sum_{n_{1} \cdots n_{r}=n} \frac{b_{1 ; \tau_{1}}\left(n_{1}\right) \cdots b_{r ; \tau_{r}}\left(n_{r}\right)}{n_{1}^{\bar{s}_{1}} \cdots n_{r}^{\bar{s}_{r}}} \\
& \times \frac{1}{M_{Q}} \sum_{q \leq Q} \sum_{\substack{\chi=\chi(\bmod q) \\
\chi \neq \chi_{0}}} \chi(m) \bar{\chi}(n) .
\end{aligned}
$$

Consider two cases $m=n$ and $m \neq n$. If $m=n$, then

$$
\begin{aligned}
\sum_{q \leq Q} \sum_{\substack{\chi=\chi(\bmod q) \\
\chi \neq \chi_{0}}} \chi(m) \bar{\chi}(n) & =\sum_{q \leq Q} \sum_{\substack{\chi=\chi(\bmod q) \\
\chi \neq \chi_{0}}}|\chi(m)|^{2}=M_{Q}-\sum_{\substack{q \mid m \\
q \leq N^{j}}}(q-2) \\
& =M_{Q}+\mathcal{O}\left(\sum_{q \leq N^{j}} q\right)=M_{Q}+\mathcal{O}\left(N^{2 j}\right)
\end{aligned}
$$

$j=2, \ldots, r$. Moreover, in view of the estimate $\sum_{d_{1} \cdots d_{j}=m} 1=\mathcal{O}_{\varepsilon}\left(m^{\varepsilon}\right), j=2, \ldots, r$, taking into account (2.7), (3.2), we deduce that, for $\left|\tau_{j}\right| \leq c$ and $s_{j} \in R_{j}$,

$$
\begin{aligned}
& \sum_{m_{1} \cdots m_{j}=m} \frac{a_{1 ; \tau_{1}}\left(m_{1}\right) \cdots a_{j ; \tau_{j}}\left(m_{j}\right)}{m_{1}^{s_{1}} \cdots m_{j}^{s_{j}}} \sum_{n_{1} \cdots n_{j}=n} \frac{b_{1 ; \tau_{1}}\left(n_{1}\right) \cdots b_{j ; \tau_{j}}\left(n_{j}\right)}{n_{1}^{\bar{s}_{1}} \cdots n_{j}^{\bar{s}_{j}}} \\
= & \mathcal{O}\left(\sum_{m_{1} \cdots m_{j}=m} \frac{d^{c_{1}}\left(m_{1}\right) \cdots d^{c_{1}}\left(m_{j}\right)}{m^{1+\delta}} \sum_{n_{1} \cdots n_{j}=m} \frac{d^{c_{1}}\left(n_{1}\right) \cdots d^{c_{1}}\left(n_{j}\right)}{n^{1+\delta}}\right)=\mathcal{O}_{\varepsilon}\left(\frac{1}{m^{2+2 \delta-\varepsilon}}\right) .
\end{aligned}
$$

Since [1] $M_{Q}=\frac{Q^{2}}{2 \log Q}+\mathcal{O}\left(\frac{Q^{2}}{\log ^{2} Q}\right)$, the latter estimate and (3.4) show that the case $m=n$ contributes to $(3.1)$

$$
\begin{aligned}
& \sum_{m=1}^{\infty} \sum_{m_{1} m_{2}=m} \frac{a_{1 ; \tau_{1}}\left(m_{1}\right) a_{2 ; \tau_{2}}\left(m_{2}\right)}{m_{1}^{s_{1}} m_{2}^{s_{2}}} \sum_{n_{1} n_{2}=m} \frac{b_{1 ; \tau_{1}}\left(n_{1}\right) b_{2 ; \tau_{2}}\left(n_{2}\right)}{n_{1}^{\bar{s}_{1}} n_{2}^{\bar{s}_{2}}}+\mathrm{o}(1), \\
& \ldots \ldots \ldots \ldots \ldots \ldots \ldots \ldots \ldots \ldots \ldots \ldots \ldots \ldots \ldots \ldots m_{r ; \tau_{r}}\left(m_{r}\right) \\
& \sum_{m=1}^{\infty} \sum_{m_{1} \cdots m_{r}=m} \frac{a_{1 ; \tau_{1}}\left(m_{1}\right) \cdots}{m_{1}^{s_{1}} \cdots m_{r}^{s_{r}}} \sum_{n_{1} \cdots n_{r}=m} \frac{b_{1 ; \tau_{1}}\left(n_{1}\right) \cdots b_{r ; \tau_{r}}\left(n_{r}\right)}{n_{1}^{\bar{s}_{1}} \cdots n_{r}^{\bar{s}_{r}}}+\mathrm{o}(1),
\end{aligned}
$$


uniformly in $\left|\tau_{j}\right| \leq c$ and $s_{j} \in R_{j}, j=1, \ldots, r$, as $Q \rightarrow \infty$.

Now suppose that $m \neq n$. Then, for $m, n \leq N^{j}, j=2, \ldots, r$, similarly, as in [3], we obtain that

$$
\sum_{q \leq Q} \sum_{\substack{\chi=\chi(\bmod q) \\ \chi \neq \chi_{0}}} \chi(m) \bar{\chi}(n)=\mathcal{O}\left(\frac{Q}{\log Q}\right) .
$$

This, (3.1), (3.3) and (3.5) show that, uniformly in $\left|\tau_{j}\right| \leq c$ and $s_{j} \in R_{j}$, $j=1, \ldots, r$,

$$
\begin{aligned}
& w_{Q}\left(\tau_{1}, \tau_{2}\right)=\sum_{m=1}^{\infty} \sum_{m_{1} m_{2}=m} \frac{a_{1 ; \tau_{1}}\left(m_{1}\right) a_{2 ; \tau_{2}}\left(m_{2}\right)}{m_{1}^{s_{1}} m_{2}^{s_{2}}} \sum_{n_{1} n_{2}=m} \frac{b_{1 ; \tau_{1}}\left(n_{1}\right) b_{2 ; \tau_{2}}\left(n_{2}\right)}{n_{1}^{\bar{s}_{1}} n_{2}^{\bar{s}_{2}}}+\mathrm{o}(1), \\
& w_{Q}\left(\tau_{1}, \ldots, \tau_{r}\right)=\sum_{m=1}^{\infty} \sum_{m_{1} \cdots m_{r}=m} \frac{a_{1 ; \tau_{1}}\left(m_{1}\right) \cdots a_{r ; \tau_{r}}\left(m_{r}\right)}{m_{1}^{s_{1}} \cdots m_{r}^{s_{r}}} \\
& \times \sum_{n_{1} \cdots n_{r}=m} \frac{b_{1 ; \tau_{1}}\left(n_{1}\right) \cdots b_{r ; \tau_{r}}\left(n_{r}\right)}{n_{1}^{\bar{s}_{1}} \cdots n_{r}^{\bar{s}_{r}}}+\mathrm{o}(1)
\end{aligned}
$$

as $Q \rightarrow \infty$.

\section{Proof of Theorem 1}

By (2.2) and (3.6), we have that the characteristic transforms of the measure $P_{Q}$ converge uniformly in $\left|\tau_{j}\right| \leq c$ and $s_{j} \in R_{j}, j=1, \ldots, r$, to the functions defined by formulae (1.1) as $Q \rightarrow \infty$. The uniform convergence ensure the continuity of the functions (1.1). Thus, the application of a continuity theorem for characteristic transforms of the measures on $\left(\mathbb{R}^{r}, \mathcal{B}\left(\mathbb{R}^{r}\right)\right)$, Theorem 3 of [4], completes the proof of Theorem 1.

\section{References}

[1] P.D.T.A. Elliott. On the distribution of the values of $L$-series in the half-plane $\sigma>\frac{1}{2}$. Indag. Math., 31(3):222-234, 1971.

[2] P.D.T.A. Elliott. On the distribution of $\arg L(s, \chi)$ in the half-plane $\sigma>\frac{1}{2}$. Acta Arith., 20:155-169, 1972.

[3] V. Garbaliauskienè, A. Laurinčikas and E. Stankus. Limit theorems for twist of L-functions of elliptic curves. Lith. Math. J., 50(2):187-197, 2010.

[4] A. Laurinčikas. Multidimensional distribution of values of multiplicative functions. Liet. Matem. Rink., 15(2):13-24, 1975.

[5] E. Stankus. The distribution of L-function. Liet. Matem. Rink., 15(3):127-134, 1975. 